\title{
Automata-Theoretic Performance Analysis Method of Soft Real-Time Systems
}

\author{
Satoshi Yamane \\ Graduate School of Natural Science, Kanazawa University, \\ Kanazawa City, Japan, Zip/Code 920-1192 \\ syamane@is.t.kanazawa-u.ac.jp
}

\begin{abstract}
Recently, the verification method of schedulability of realtime operating systems using timed automata have been developed. On the other hand, as soft real-time systems such as distributed systems and multimedia systems have been increasing, it is important to design soft real-time systems. Especially, performance analysis methods are important for soft real-time systems. In this paper, we develop the automata-theoretic performance analysis method of soft real-time systems by extending the verification method of schedulability of hard realtime systems using utility functions.
\end{abstract}

\section{Introduction}

Real-time systems are of vital economic importance and are literally becoming ubiquitous. They have already become an integral component of safety critical systems involving aviation, telecommunications, and process control applications. It is important to formally specify and verify real-time systems. Real-time systems are characterized as those where the correctness of applications depends not only on the correctness of the logical computation being performed, but also on the time at which the results are produced [1]. Real-time systems are classified into hard real-time systems and soft real-time systems [2]. Hard real-time systems do not tolerate any missed deadlines. This requires that the task scheduling is based on worst-case task execution time estimates. This leads to inefficient utilization of resources, particularly when tasks normally require much less time to complete execution than the worst case estimates, and the worst case behavior is very rare. On the other hand, in soft real-time systems, where some missed deadlines can be tolerated, it is not necessary to use worst case execution time estimates. The probability that all tasks in the system complete by a specified deadline can be increased by allowing a small amount of slack times. The slack time is to tolerate the failure of some tasks completing within their assigned execution times. Thus trade-off between the guarantee that all tasks meet their deadlines and the cost of such guarantees can be made.

In hard real-time systems, it is important to verify whether worst-case task execution time is less than deadline or not. This verification is called schedulability [1]. On the other hand, in soft real-time systems, the performance of a 
scheduling algorithm can be measured by accumulating the values of the task utility functions computed at their complete time 3. Today, timed automaton [4] is the standard tool for specifying and verifying real-time systems by modelchecking methods [5,6]. Moreover, recently, the schedulability checking problem can be solved using extended timed automata by Wang Yi [7,8]. Before Wang Yi's study [8], the preemptive schedulability checking problem has been able to be solved using only stopwatch automata [9, 10, 11, 12, 13.

On the other hand, in soft real-time systems, the performance of a scheduling algorithm has been measured by accumulating the values of the task utility functions computed at their complete time or determining lower bounds on the frequency of missed deadlines as follows. (1)In 1985, E.D. Jensen has used value functions to describe the performance of existing process scheduling algorithms 14. (2)In 1995, B.Kao has studied the performance of distributed soft real-time systems that use standard components with various scheduling algorithms and have suggested ways to improve them [15]. (3)In 1994, K.M. Kavi has studied a simple model that combines the failure to meet deadlines with hardware/software failures in computing the reachability of a real-time systems. They have defined the performability as the probability of meeting deadlines by real-time tasks in the presence of hardware and software failures. (4)In 1999, M.K. Gardner and J.W.S. Liu have studied an analysis technique by which a lower bound on the percentage of deadlines that a periodic task meets is determined and compare the lower bound with simulation results for an example system [17].

On the other hand, some researchers have extended existing languages with probabilities such as probabilistic process algebra [18, probabilistic timed automaton with continuous probabilities [19,21], probabilistic timed automaton with discrete probabilities [20]. But they have not studied performance analysis.

In this paper, we propose an automata-theoretic performance analysis method that combines timed automata with utility functions. More practically speaking, we extend Wang Yi's timed automata [8] with utility functions. While existing studies have been based on simulation methods, our proposed method is based on mathematical model. Using our proposed method, we can formally evaluate schedulers of soft real-time systems such as multimedia systems and distributed systems. Our proposed method is different from Wang Yi's method [8] in the following two important points:

1. In Wang Yi's method, task is characterized as the execution time and the deadline. On the other hand, in our proposed method, task is characterized as the execution time, the deadline and the utility function.

2. In Wang Yi's method, the schedulability problem can be transformed to a reachability problem for timed automata. On the other hand, in our proposed method, the performance analysis can be transformed to a brute force search for timed computation tree of timed automata and a calculation of the value of every state.

The paper is organized as follows: In section 2, we propose extended timed automata for performance analysis of soft real-time systems. In section 3, we propose our automata-theoretic performance analysis method. In section 4 , we 
propose algorithms of our automata-theoretic performance analysis. Finally, in section 5, we present conclusions.

\section{Timed Automata of Performance Analysis}

In this paper, we propose extended timed automata for performance analysis of soft real-time systems.

\subsection{Soft Real-Time Systems}

First we define soft real-time systems. In soft real-time systems, where some missed deadlines can be tolerated, it is not necessary to use worst case execution time estimates. The probability that all tasks in the system complete by a specified deadline can be increased by allowing a small amount of slack times. According to the probability, the value of a task decreases. The task importance can be better described by an utility function. Figure 1 illustrates some utility functions that can be associated with tasks in order to describe their importance. A hard task contributes to a value only if it completes within its deadline $D$, and since a deadline miss would jeopardize the behavior of the whole system, the value $v(t)$ after its deadline $D$ can be considered minus infinity in many situations. A task with a soft deadline, instead, can still give a value $v(t)$ to the system if executed after its deadline $D$, although this value may decrease with time.

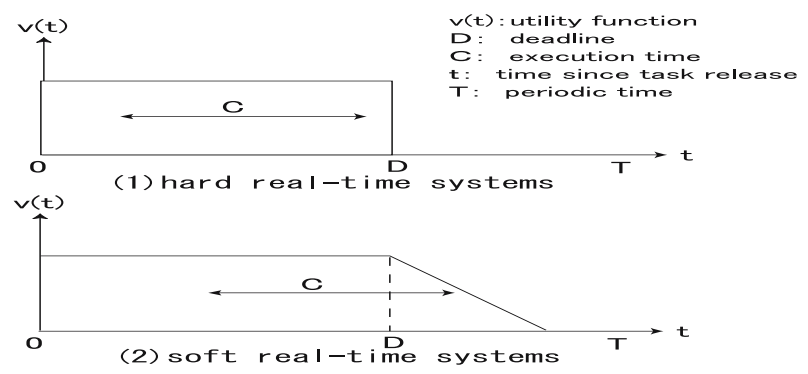

Fig. 1. Utility function that can be associated to a task to describe its importance

\subsection{Syntax of Extended Timed Automata}

First, we define task as follows.

\section{Definition 1 (Task)}

Let $\mathcal{P}$ ranged over by $P$ and $Q, R$ etc, denote a finite set of task types. A task type may have different instances that are copies of the same program with different inputs. Each task $P$ is characterized as a pair of natural numbers denoted $P\left(D_{P}, C_{P}, v_{P}\left(t_{P}\right)\right)$ with $C_{P} \leq D_{P}$, where $C_{P}$ is the execution time(or computation time) of $P, D_{P}$ is the deadline for $P, v_{P}\left(t_{P}\right)$ is the utility function. 
Next, we introduce a preliminary.

\section{Definition 2 (Preliminary)}

We introduce a preliminary.

1. Act is a finite alphabet for actions. Let be Act $=\{a, b, c, \ldots\}$.

2. $\mathcal{C}$ is a finite set of real-valued variables. Let be $\mathcal{C}=\left\{x_{1}, x_{2}, x_{3}, \ldots\right\}$.

3. We use $\mathcal{B}(\mathcal{C})$ ranged over by $g$ to denote the set of conjunctive formulas of atomic constraints in the form: $x_{i} \sim d_{i}$ or $x_{i}-x_{j} \sim d_{i j}$, where $x_{i}, x_{j} \in \mathcal{C}$, $\sim \in\{\leq,<, \geq,>\}, d_{i}, d_{i j}$ are natural numbers.

Next, we define extended timed automaton.

\section{Definition 3 (Syntax of extended timed automaton)}

An extended timed automaton $\mathbf{A}$ is a tuple $\left(N, l_{0}, E, I, M\right)$, where

1. $N$ is a finite set of locations ranged over by $l, m, n$

2. $l_{0} \in N$ is the initial location

3. $E \subseteq N \times \mathcal{B}(\mathcal{C}) \times$ Act $\times 2^{\mathcal{C}} \times N$ is the set of edges

4. $I: N \hookrightarrow \mathcal{B}(\mathcal{C})$ is a function assigning each location with a clock constraint

5. $M: N \hookrightarrow \mathcal{P}$ is a partial function assigning locations with tasks

Next we show some simple example of a task model.

\section{Example 1 (A simple example of a task model)}

Tasks are classified into periodic tasks, aperiodic tasks, sporadic tasks [22]. In the periodic task model, each computation or data transmission that is executed repeatly at regular or semiregular time intervals in order to provide a function of the system on a continuing basis is modeled as a periodic task. On the other hand, a task is aperiodic if the jobs in the task have either soft deadlines or no deadlines. Moreover, a task is sporadic if the jobs in the task are sporadically released. We show examples of periodic tasks, aperiodic tasks, sporadic tasks, and specify them using extended timed automata in Figure 2.

\subsection{Semantics of Extended Timed Automata}

Next, we define semantics of extended timed automata.

Extended timed automata perform two types of transitions. Delay transitions correspond to the execution of running tasks with high priority and idling for the other tasks waiting to run. Discrete transitions correspond to the arrival of new task instances.

First, we define semantic states as follows:

\section{Definition 4 (Semantic states of timed automaton)}

We represent the values of clocks as functions from $C$ to the non-negative reals $\mathbf{R} . \mathcal{V}$ denotes the set of assignments of $C$. A semantic state of an extended timed automaton is a triple $(l, u, q)$, where $l \in N$ is the current node, $u \in \mathcal{V}$ denotes the current values of clocks, and $q$ is the current task queue. We assume that the task queue takes the form: 


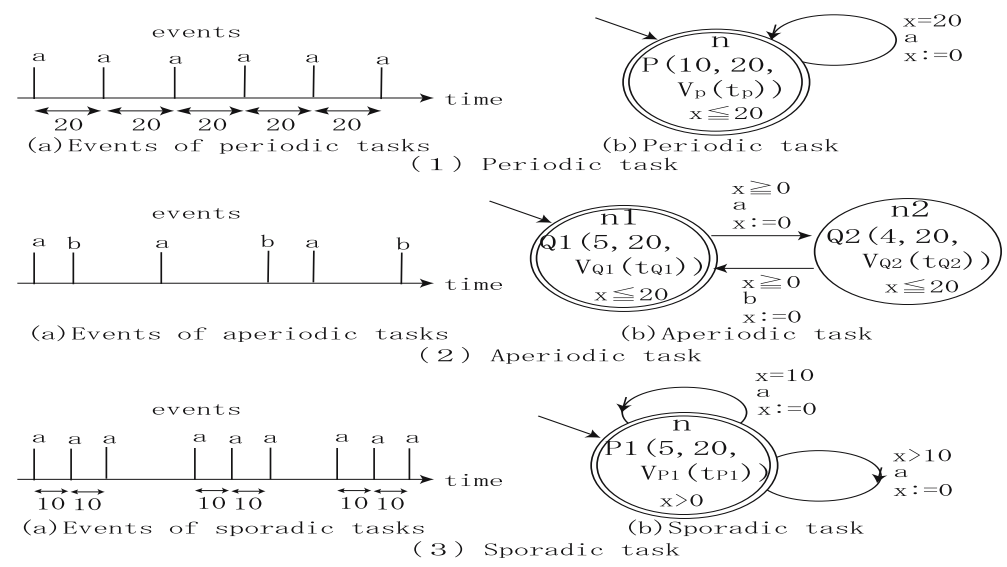

Fig. 2. Examples of periodic tasks, aperiodic tasks, sporadic tasks

$$
\left[P_{1}\left(c_{P_{1}}, d_{P_{1}}, v_{P_{1}}\left(t_{P_{1}}\right)\right), \ldots \ldots, P_{n}\left(c_{P_{n}}, d_{P_{n}}, v_{P_{n}}\left(t_{P_{n}}\right)\right)\right],
$$

where $P_{i}\left(c_{P_{i}}, d_{P_{i}}, v_{P_{i}}\left(t_{P_{i}}\right)\right)$ denotes a released instance of task type $P_{i}$ with remaining computing time $c_{P_{i}}$, relative deadline $d_{P_{i}}$ and value $v_{P_{i}}\left(t_{P_{i}}\right)$. Here $t_{P_{i}}$ denotes the elapsed time since the release of $P_{i}$.

A scheduling strategy Sch such as FPS (Fixed Priority Scheduling) and EDF(Earliest Deadline First) is a sorting function which changes the ordering of the task queue elements according to the task parameters. A delay transition with $t$ time units is to execute the task in the first position of the queue with $t$ time units. Thus the delay transition will decrease the computing time of the first task by $t$ and increase the elapsed time $t_{P}$ by $t$, and compute $v_{P}\left(t_{P}\right)$. If the computation time becomes 0 , the task should be removed from the queue. We define the followings:

1. Sch is a sorting function which changes the ordering of the task queue elements.

For example, $\operatorname{EDF}\left(\left[P\left(3.1,10, v_{p}\left(t_{p}\right)\right), Q\left(4,5.3, v_{q}\left(t_{q}\right)\right)\right]\right)=\left[Q\left(4,5.3, v_{q}\left(t_{q}\right)\right)\right.$, $\left.P\left(3.1,10, v_{p}\left(t_{p}\right)\right)\right]$.

2. Run is a function which given a real number $t$ and a task $q$ returns the resulted task queue after $t$ time units of execution according to available computing resources.

For example, let $q=\left[Q\left(4,5, v_{q}\left(t_{q}\right)\right), P\left(3,10, v_{p}\left(t_{p}\right)\right)\right]$. Then $\operatorname{Run}(q, 6)=$ $\left[P\left(1,4, v_{p}\left(t_{p}+6\right)\right)\right]$ and, the value of $Q$ is $v_{q}\left(t_{q}+4\right)$.

Next we define $u \models g, u+t, u[r \mapsto 0], x:=x-c$ in order to define the operational semantics of timed automaton.

1. We use $u=g$ to denote that the clock assignment $u$ satisfies the constraint $g$.

2. We use $u+t$ to denote the clock assignment which maps each $x$ to the value $u(x)+t$. 
3. We use $u[r \mapsto 0]$ for $r \subseteq \mathcal{C}$ to denote the clock assignment which each clock in $r$ to 0 and agrees with $u$ for the other clocks.

4. We use $x:=x-c$ to denote the subtraction of $c$ from $x$.

We define the operational semantics of extended time automata as follows:

\section{Definition 5 (Operational semantics)}

Given a scheduling strategy $\mathbf{S c h}$, the semantics of an extended timed automaton $\mathbf{A}=\left(N, l_{0}, E, I, M\right)$ with an initial state $\left(l_{0}, u_{0}, q_{0}\right)$ is a transition system defined by the following rules:

1. discrete transition:

$(l, u, q) \stackrel{g, a, r}{\longrightarrow} S c h(m, u[r \mapsto 0], \mathbf{S c h}(M(m):: q))$ if $l \stackrel{g, a, r}{\longrightarrow} m$ and $u \models g$

2. delay transition:

$(l, u, q) \stackrel{t}{\longrightarrow} S c h(l, u+t, \boldsymbol{R u n}(q, t))$ if $(u+t) \models I(l)$,

where $t$ is assigned to the maximum delay as we execute maximum numbers of tasks.

Here $M(m):: q$ denotes the queue $q$ with $M(m)$ inserted into it.

We show an example as follows:

\section{Example 2 (Example of behavior of tasks)}

First we show an example of a task model. $P_{1}$ and $P_{2}$ are periodic tasks, and $Q_{1}$ and $Q_{2}$ are triggered by events. First, a task $Q_{1}$ is triggered in node $m_{1}$, The automaton models a system starting in node $m_{1}$, which moves to node $n_{1}$ by event $a_{1}$ at 10 time, which triggers the task $P_{1}$. Moreover, the system periodically triggers the task $P_{1}$ at 20 time in node $n_{1}$. After that, the system moves to node $m_{2}$ by event $b_{2}$ at any time, which triggers the $Q_{2}$.

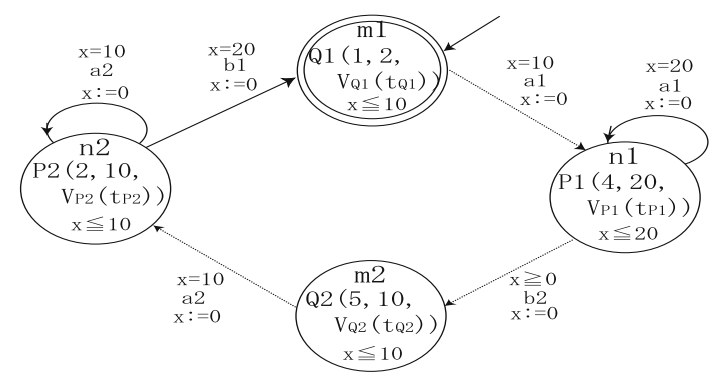

Fig. 3. Example of an extended timed automaton

Assume that preemptive earliest deadline (EDF) is used to schedule the task queue. Then the automaton with initial state may demonstrate the following sequence of typical transitions: 


$$
\begin{gathered}
\left(m_{1},[x=0],\left[Q_{1}\left(1,2, v_{Q_{1}}(0)\right]\right)\right. \\
\stackrel{1}{\longrightarrow} S c h\left(m_{1},[x=1],\left[Q_{1}\left(0,1, v_{Q_{1}}(1)\right]\right)=\left(m_{1},[x=1],[]\right)\right. \\
\stackrel{9}{\longrightarrow} S c h\left(m_{1},[x=10],[]\right) \\
\stackrel{a_{1}}{\longrightarrow} S c h\left(n_{1},[x=0],\left[P_{1}\left(4,20, v_{P_{1}}(0)\right]\right)\right. \\
\stackrel{4}{\longrightarrow} S c h\left(n_{1},[x=4],\left[P_{1}\left(0,16, v_{P_{1}}(4)\right]\right)=\left(n_{1},[x=4],[]\right)\right.
\end{gathered}
$$

\section{Automata-Theoretic Performance Analysis Method}

In this section, we propose an automata-theoretic performance analysis method of soft real-time systems. In 2002, Wang Yi and his colleagues have shown that schedulability checking problem based on timed automata is decidable [7,8]. In this paper, we propose an automata-theoretic performance analysis method of soft real-time systems by extending Wang Yi's method. In Wang Yi's method, the schedulability problem can be transformed to a reachability problem for timed automata. On the other hand, in our proposed method, the performance analysis can be transformed to a brute force search for timed computation tree of timed automata and a calculation of the value of every state. In general, as real-time systems is nondeterministic, we must search for timed computation tree [5].

We realize an automata-theoretic performance analysis method by the followings:

For a given timed automaton, we construct a timed computation tree with root state $\left(l_{0}, u_{0}, q_{0}\right)$ and other states such as $\left(l_{k}, u_{k}, q_{k}\right)(k \geq 1)$. At the same time, we compute the value of $\left(l_{k}, u_{k}, q_{k}\right)$. Finally, we sum up the values per each deterministic behavior.

First, we define the notion of timed sequence of extended timed automaton.

\section{Definition 6 (Timed sequence)}

We define the notion of timed sequence of extended timed automaton as follows:

For an automaton with initial state $\left(l_{0}, u_{0}, q_{0}\right)$, timed sequence is as follows:

$$
\omega=\left(l_{0}, u_{0}, q_{0}\right) \stackrel{t_{0}}{\longrightarrow} \ldots \stackrel{g_{i-1}, a_{i-1}, r_{i-1}}{\longrightarrow}(l, u, q) \stackrel{t_{i}}{\longrightarrow} \ldots
$$

In general, one extended timed automaton has a number of timed sequences.

Next, we define the notion of reachability as for extended timed automaton.

\section{Definition 7 (Reachability)}

We define the notion of reachability as for extended timed automaton as follows:

For an automaton with initial state $\left(l_{0}, u_{0}, q_{0}\right),(l, u, q)$ is reachable

$$
\begin{gathered}
\omega=\left(l_{0}, u_{0}, q_{0}\right) \stackrel{t_{0}}{\longrightarrow} \ldots \stackrel{\text { iff }}{\longrightarrow} \stackrel{g_{i-1}, a_{i-1}, r_{i-1}}{\longrightarrow}(l, u, q) \\
\omega=\left(l_{0}, u_{0}, q_{0}\right) \stackrel{t_{0}}{\longrightarrow} \ldots \stackrel{t_{i-1}}{\longrightarrow}(l, u, q) .
\end{gathered}
$$


Next we define an automata-theoretic performance analysis method of soft real-time systems.

\section{Definition 8 (An Automata-Theoretic Performance Analysis Method)} For a given timed automaton, we construct a timed computation tree with root state $\left(l_{0}, u_{0}, q_{0}\right)$ and other states such as $\left(l_{k}, u_{k}, q_{k}\right)(k \geq 1)$. At the same time, we compute the value of $\left(l_{k}, u_{k}, q_{k}\right)(k=0,1,2, \ldots)$ as follows:

where

$$
q_{k}=\left[P_{1}{ }^{k}\left(c_{P_{1}}{ }^{k}, d_{P_{1}}{ }^{k}, v_{P_{1}}\left(t_{P_{1}}{ }^{k}\right)\right), \ldots, P_{n}{ }^{k}\left(c_{P_{n}}{ }^{k}, d_{P_{n}}{ }^{k}, v_{P_{n}}\left(t_{P_{n}}{ }^{k}\right)\right)\right] .
$$

1. For each deterministic behavior, from each initial state, we construct the timed sequence consisting of the set of semantic states, and compute values as follows:

(a) Case of $t_{P_{i}}{ }^{k}>T_{P_{i}}$ :

System error occurs,

where $t_{P_{i}}{ }^{k}$ is the elapsed time since release of $P_{i}$, and $T_{P_{i}}$ is the period or the constraint of $P_{i}$.

(b) Case of $t_{P_{i}}{ }^{k} \leq T_{P_{i}}$ :

We compute the sum of values as follows:

For $\forall i$, we compute the value using $v_{P_{i}}\left(t_{P_{i}}{ }^{k}\right)$ at $c_{i}{ }^{k}=0$ :

$$
\sum_{i=1}^{n} v_{P_{i}}\left(t_{P_{i}}{ }^{k}\right)
$$

2. We repeat the above procedure 1., and compute the set of sums. For example, the sum is $\sum_{i=1}^{n} v_{P_{i}}\left(t_{P_{i}}{ }^{k}\right)$. Finally, the number of sums, which we have computed, is equal to the number of nondeterministic behaviors. Each deterministic behavior has each sum.

Finally, we mention that an automata-theoretic performance analysis method is decidable.

Theorem 1 (Decidability of an automata-theoretic performance analysis) The problem of computing an automata-theoretic performance analysis for extended timed automaton is decidable.

Proof 1. Our extended timed automaton is the class of bounded timed automaton with subtraction [8]. Thus the problem of computing performance analysis for extended timed automaton is decidable.

\section{Implementation}

In this section, we show the implementation of an automata-theoretic performance analysis method.

We compute the performance in Definition 8 according to semantic transitions in Definition 5. In this performance analysis method, it is important 
to compare elapsed time with remaining computing time, and compute values if remaining computing time becomes zero. We use DBMs(Difference Bounds Matrices) 223, 24] in order to compute remaining computing time.

First, we define DBMs as follows.

\section{Definition 9 (DBMs)}

Suppose the extended timed automaton has $n$ clocks, $x_{1}, \ldots, x_{n}$. Then a clock zone is represented by a $(n+1) \times(n+1)$ matrix $D$. For each $i$, the entry $D_{i 0}$ gives an upper bound on the clock $x_{i}$, and the entry $D_{0 i}$ gives a lower bound on the clock $x_{i}$. For every pair $i, j$, the entry $D_{i j}$ gives an upper bound on the difference of the clocks $x_{i}$ and $x_{j}$. To distinguish between a strict and a nonstrict bound (i.e. to distinguish between constraints such as $x<2$ and $x \leq 2$ ), and allow for the possibility of absence of a bound, define the bounds-domain to be $\mathbf{Z} \times\{<, \leq\} \cup\{\infty\}$. The constant $\infty$ denotes the absence of a bound, the bound $(c, \leq)$ denotes $\leq c$, the bound $(c,<)$ denotes $<c$.

Next we define Operations of DBMs.

\section{Definition 10 (Operations of DBMs)}

In this paper, we use the following operations:

\section{Intersection:}

The intersection of two DBMs D and DI can easily be computed from two $D B M s$. The intersection $D B M s$ DIII $=D \cap D \prime$ is the canonical form of DBMs DII, which is computed by the followings:

$$
D_{i j} \prime \prime=\min \left\{D_{i j}, D_{i j}\right\}
$$

2. Time successor:

$D \nearrow$ is obtained from $D$ by removing all inequalities that place upper bounds on the absolute values of the clocks, i.e. inequalities of the form $x_{i}-x_{0} \leq d_{i 0}$ or $x_{i}-x_{0}<d_{i 0}$.

3. Reset:

$D[r:=0]$ is obtained from $D$ by first ignoring all constraints on variables in $r$, and then taking the subset for which all variables in $r$ equal to zero.

Next we define the method of computing task queue.

\section{Definition 11 (Computing task queue by DBMs)}

For state $\left(l_{k}, u_{k}, q_{k}\right)(k=0,1,2, \ldots)$, we define the method of computing such task queue as

$$
q_{k}=\left[P_{1}^{k}\left(c_{P_{1}}{ }^{k}, d_{P_{1}}{ }^{k}, v_{P_{1}}\left(t_{P_{1}}{ }^{k}\right)\right), \ldots, P_{n}{ }^{k}\left(c_{P_{n}}{ }^{k}, d_{P_{n}}{ }^{k}, v_{P_{n}}\left(t_{P_{n}}{ }^{k}\right)\right)\right] .
$$

We compute the task queue by dividing transitions into delay and discrete transition.

\section{Delay transition:}

First we compute the elapsed time in the state $\left(l_{k}, u_{k}, q_{k}\right)$. Assume that DBMs is $D$ in the entrance of the state, where all the clocks are zero in an initial state. Here we introduce the elapsed timer $t_{l_{k}}$. D becomes D' by the fact that $t_{l_{k}}$ is 
added to $D$. As the minimum clock is assigned to $t_{l_{k}}$, the following entries are added to $D$.

$$
t_{l_{k}}-x_{0} \leq 0, t_{l_{k}}-x_{1} \leq 0, \ldots, t_{l_{k}}-x_{n} \leq 0, x_{0}-t_{l_{k}} \leq 0
$$

We compute the elapsed time in state $\left(l_{k}, u_{k}, q_{k}\right)$ based on $D \prime$.

$\left(\left(\left(D \prime \cap D_{I\left(l_{k}\right)}\right) \nearrow\right) \cap D_{I\left(l_{k}\right)}\right) \cap D_{g}$,

where $D_{I\left(l_{k}\right)}$ is DBMs obtained from clock constraint in node $l_{k}, D_{g}$ is DBMs obtained from $\mathrm{g}$.

Now we compute $\left(l_{k}, u_{k}+t_{l_{k}}, \boldsymbol{\operatorname { R u n }}\left(q_{k}, t_{l_{k}}\right)\right)$ using $t_{l_{k}}$.

In the followings, we compute $\operatorname{Run}\left(q_{k}, t_{l_{k}}\right)$ by dividing $t_{l_{k}}$ into various cases. $t_{l_{k}}$ is divided into $t_{l_{k}} \leq d_{l_{k}}, t_{l_{k}}=d_{l_{k}}, t_{l_{k}}<d_{l_{k}}, t_{l_{k}}>d_{l_{k}}, t_{l_{k}} \geq d_{l_{k}}$. Moreover, according to relations between $d_{l_{k}}$ and $c_{P_{1}}{ }^{k}, c_{P_{1}}{ }^{k}$ is divided into $c_{P_{1}}{ }^{k} \leq d_{l_{k}}$, $c_{P_{1}}{ }^{k}=d_{l_{k}}, c_{P_{1}}{ }^{k}<d_{l_{k}}, c_{P_{1}}{ }^{k}>d_{l_{k}}, c_{P_{1}}{ }^{k} \geq d_{l_{k}}$.

We show these cases by the Figure 4 .

(1) $\mathrm{t} 1 \mathrm{k} \leqq \mathrm{d} 1 \mathrm{k}$

(2) $t_{1 k}<d_{1 k}$

(3) $\mathrm{t}_{1 \mathrm{k}}=\mathrm{d} 1 \mathrm{k}$

(4) $t_{1 k} \geqq d_{1 k}$

(5) $t_{1 k}>\mathrm{d} 1 \mathrm{k}$
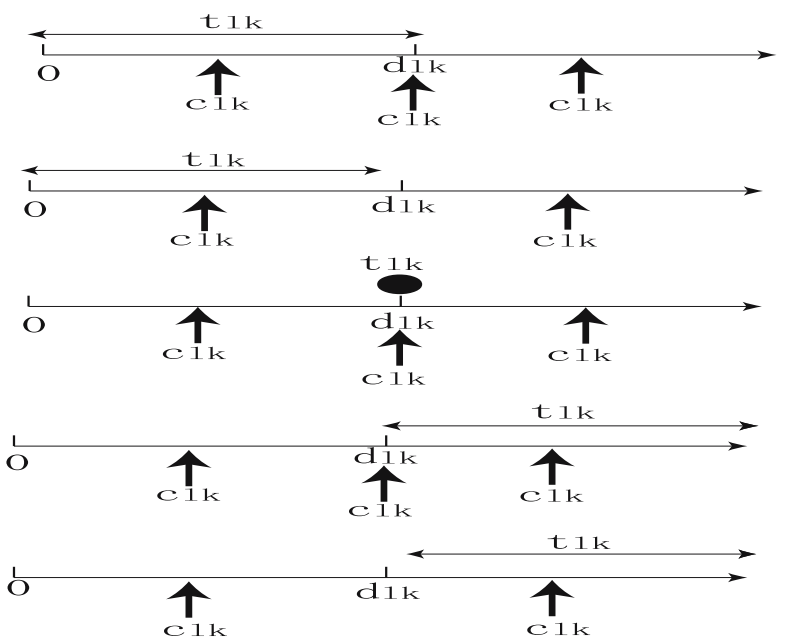

Fig. 4. Cases of $t_{l_{k}}$

In the followings, we compute $\operatorname{Run}\left(q_{k}, t_{l_{k}}\right)$ according to the above cases. Here task $P_{i}$ has an error, if $t_{l_{k}}$ is greater than $T_{P_{i}}$.

1. $t_{l_{k}} \leq d_{l_{k}}$ :

Then we consider three cases such as $c_{P_{i}}{ }^{k}<d_{l_{k}}, c_{P_{i}}{ }^{k}=d_{l_{k}}, c_{P_{i}}{ }^{k}>d_{l_{k}}$.

(a) $c_{P_{1}}{ }^{k}<d_{l_{k}}$

In this case, task queue is as follows:

$$
\begin{aligned}
\operatorname{Run} & \left(q_{k}, t_{l_{k}}\right)=[ \\
& P_{1}{ }^{k}\left(0, d_{P_{1}}{ }^{k}-c_{P_{1}}{ }^{k}, v_{P_{1}}\left(t_{P_{1}}{ }^{k}+c_{P_{1}}{ }^{k}\right)\right), \\
& \left.\ldots \ldots \ldots,{ }^{k}{ }^{k}-\left(c_{P_{1}}{ }^{k}+\ldots+c_{P_{i-1}}{ }^{k}\right), v_{P_{i}}\left(t_{P_{i}}{ }^{k}+\left(c_{P_{1}}{ }^{k}+\ldots+c_{P_{i-1}}{ }^{k}\right)\right)\right),
\end{aligned}
$$




$$
\begin{aligned}
& P_{i+1}{ }^{k}\left(c_{P_{i+1}}{ }^{k}-\left(d_{l_{k}}-\left(c_{P_{1}}{ }^{k}+\ldots+c_{P_{i}}{ }^{k}\right)\right), d_{P_{i}}{ }^{k}-d_{l_{k}}, v_{P_{i}}\left(t_{P_{i+1}}{ }^{k}+d_{l_{k}}\right)\right), \\
& \ldots \ldots \ldots ., \\
& \left.P_{n}{ }^{k}\left(c_{P_{n}}{ }^{k}, d_{P_{n}}{ }^{k}-d_{l_{k}}, v_{P_{n}}\left(t_{P_{n}}{ }^{k}+d_{l_{k}}\right)\right)\right] \text {, where } t_{l_{k}}=d_{l_{k}} .
\end{aligned}
$$

Therefore we compute the value as follows:

(b) $c_{P_{1}}{ }^{k}=d_{l_{k}}$ :

$$
\left.\left.v_{P_{1}}\left(t_{P_{1}}{ }^{k}+c_{P_{1}}{ }^{k}\right)\right)+v_{P_{2}}\left(t_{P_{2}}{ }^{k}+c_{P_{1}}{ }^{k}+c_{P_{2}}{ }^{k}\right)\right)+\ldots
$$

In this case, task queue is as follows:

$\operatorname{Run}\left(q_{k}, t_{l_{k}}\right)=$

$$
\begin{aligned}
& P_{1}^{k}\left(0, d_{P_{1}}{ }^{k}-c_{P_{1}}{ }^{k}, v_{P_{1}}\left(t_{P_{1}}{ }^{k}+c_{P_{1}}{ }^{k}\right)\right), \\
& P_{2}{ }^{k}\left(c_{P_{2}}{ }^{k}, d_{P_{2}}{ }^{k}-c_{P_{1}}{ }^{k}, v_{P_{2}}\left(t_{P_{2}}{ }^{k}+d_{l_{k}}\right)\right), \\
& \ldots \ldots \ldots ., \\
& \left.P_{n}{ }^{k}\left(c_{P_{n}}{ }^{k}, d_{P_{n}}{ }^{k}, v_{P_{n}}\left(t_{P_{n}}{ }^{k}+d_{l_{k}}\right)\right)\right], \text { where } t_{l_{k}}=d_{l_{k}} .
\end{aligned}
$$

Therefore we compute the value as follows:

(c) $c_{P_{1}}{ }^{k}>d_{l_{k}}$ :

$$
\left.v_{P_{1}}\left(t_{P_{1}}^{k}+c_{P_{1}}^{k}\right)\right)
$$

In this case, task queue is as follows:

As $\operatorname{Run}\left(q_{k}, t_{l_{k}}\right)=[$

$$
\begin{aligned}
& \quad P_{1}{ }^{k}\left(c_{P_{1}}{ }^{k}-d_{l_{k}}, d_{P_{1}}{ }^{k}-d_{l_{k}}, v_{P_{1}}\left(t_{P_{1}}{ }^{k}+d_{l_{k}}\right),\right. \\
& P_{2}{ }^{k}\left(c_{P_{2}}{ }^{k}, d_{P_{2}}{ }^{k}, v_{P_{2}}\left(t_{P_{2}}{ }^{k}+d_{l_{k}}{ }^{k}\right)\right), \\
& \ldots \ldots \ldots, \\
& \left.P_{n}{ }^{k}\left(c_{P_{n}}{ }^{k}, d_{P_{n}}{ }^{k}, v_{P_{n}}\left(t_{P_{n}}{ }^{k}+d_{l_{k}}\right)\right)\right] . \\
& \text { we can not compute the value, where } t_{l_{k}}=d_{l_{k}} .
\end{aligned}
$$

2. $t_{l_{k}}<d_{l_{k}}$ :

In this case, we consider two cases such as $c_{P_{i}}{ }^{k}<d_{l_{k}}, c_{P_{i}}{ }^{k} \geq d_{l_{k}}$.

(a) $c_{P_{1}}{ }^{k}<d_{l_{k}}$ :

In this case, task queue is as follows:

$\operatorname{Run}\left(q_{k}, t_{l_{k}}\right)=[$

$$
\begin{aligned}
& \quad P_{1}^{k}\left(0, d_{P_{1}}{ }^{k}-c_{P_{1}}{ }^{k}, v_{P_{1}}\left(t_{P_{1}}{ }^{k}+c_{P_{1}}{ }^{k}\right)\right), \\
& \left.\ldots \ldots \ldots .,{ }^{k}-\left(c_{P_{1}}{ }^{k}+\ldots+c_{P_{i-1}}{ }^{k}\right), v_{P_{i}}\left(t_{P_{i}}{ }^{k}+\left(c_{P_{1}}{ }^{k}+\ldots+c_{P_{i-1}}{ }^{k}\right)\right)\right), \\
& P_{i}^{k}\left(0, d_{P_{i}}{ }^{k}{ }^{k}-\left(d_{l_{k}}{ }^{k}-\left(c_{P_{1}}{ }^{k}+\ldots+c_{P_{i}}{ }^{k}\right)\right), d_{P_{i}}{ }^{k}-d_{l_{k}}{ }^{k}, v_{P_{i}}\left(t_{P_{i+1}}+\right.\right. \\
& \\
& \left.P_{i+1}{ }^{k}\left(c_{P_{i+1}}{ }_{d_{l_{k}}}{ }^{-}\right)\right),
\end{aligned}
$$

$\left.P_{n}^{k}\left(c_{P_{n}}{ }^{k}, d_{P_{n}}{ }^{k}-d_{l_{k}}{ }^{-}, v_{P_{n}}\left(t_{P_{n}}{ }^{k}+d_{l_{k}}{ }^{-}\right)\right)\right]$, where $d_{l_{k}}{ }^{-}$is almost equal to $d_{l_{k}}$ and less than $d_{l_{k}}$.

Here $t_{l_{k}}=d_{l_{k}}{ }^{-}$.

Therefore we compute the value as follows:

(b) $c_{P_{1}}{ }^{k} \geq d_{l_{k}}$

$$
\left.\left.v_{P_{1}}\left(t_{P_{1}}{ }^{k}+c_{P_{1}}{ }^{k}\right)\right)+v_{P_{2}}\left(t_{P_{2}}{ }^{k}+c_{P_{1}}{ }^{k}+c_{P_{2}}{ }^{k}\right)\right)+\ldots
$$

In this case, task queue is as follows:

As $\operatorname{Run}\left(q_{k}, t_{l_{k}}\right)=[$

$$
\begin{aligned}
& P_{1}{ }^{k}\left(c_{P_{1}}{ }^{k}-d_{l_{k}}{ }^{-}, d_{P_{1}}{ }^{k}-d_{l_{k}}{ }^{-}, v_{P_{1}}\left(t_{P_{1}}{ }^{k}+d_{l_{k}}{ }^{-}\right),\right. \\
& P_{2}{ }^{k}\left(c_{P_{2}}{ }^{k}, d_{P_{2}}{ }^{k}-d_{l_{k}}{ }^{-}, v_{P_{2}}\left(t_{P_{2}}{ }^{k}+d_{l_{k}}{ }^{-}\right)\right), \\
& \ldots \ldots \ldots ., \\
& \left.P_{n}{ }^{k}\left(c_{P_{n}}{ }^{k}, d_{P_{n}}{ }^{k}-d_{l_{k}}{ }^{-}, v_{P_{n}}\left(t_{P_{n}}{ }^{k}+d_{l_{k}}{ }^{-}\right)\right)\right] \text {, we can not compute the }
\end{aligned}
$$
value.

Here $t_{l_{k}}=d_{l_{k}}{ }^{-}$. 
3. $t_{l_{k}}=d_{l_{k}}$ :

In this case, we consider three cases such as $c_{P_{i}}{ }^{k}<d_{l_{k}}, c_{P_{i}}{ }^{k}=d_{l_{k}}, c_{P_{i}}{ }^{k}>$ $d_{l_{k}}$.

(a) $c_{P_{1}}{ }^{k}<d_{l_{k}}$ :

In this case, task queue is as follows:

$\operatorname{Run}\left(q_{k}, t_{l_{k}}\right)=[$

$$
\begin{aligned}
& P_{1}^{k}\left(0, d_{P_{1}}{ }^{k}-c_{P_{1}}{ }^{k}, v_{P_{1}}\left(t_{P_{1}}{ }^{k}+c_{P_{1}}{ }^{k}\right)\right), \\
& \ldots \ldots \ldots, \ldots, \\
& P_{i}{ }^{k}\left(0, d_{P_{i}}{ }^{k}-\left(c_{P_{1}}{ }^{k}+\ldots+c_{P_{i-1}}{ }^{k}\right), v_{P_{i}}\left(t_{P_{i}}{ }^{k}+\left(c_{P_{1}}{ }^{k}+\ldots+c_{P_{i-1}}{ }^{k}\right)\right)\right), \\
& P_{i+1}{ }^{k}\left(c_{P_{i+1}}{ }^{k}-\left(d_{l_{k}}-\left(c_{P_{1}}{ }^{k}+\ldots+c_{P_{i}}{ }^{k}\right)\right), d_{P_{i}}{ }^{k}-d_{l_{k}}, v_{P_{i}}\left(t_{P_{i+1}}{ }^{k}+d_{l_{k}}\right)\right), \\
& \ldots \ldots \ldots ., \\
& \left.P_{n}{ }^{k}\left(c_{P_{n}}{ }^{k}, d_{P_{n}}{ }^{k}-d_{l_{k}}, v_{P_{n}}\left(t_{P_{n}}{ }^{k}+d_{l_{k}}\right)\right)\right] .
\end{aligned}
$$

Here $t_{l_{k}}=d_{l_{k}}$.

Therefore we compute the value as follows:

(b) $c_{P_{1}}{ }^{k}=d_{l_{k}}$

$$
\left.\left.v_{P_{1}}\left(t_{P_{1}}{ }^{k}+c_{P_{1}}{ }^{k}\right)\right)+v_{P_{2}}\left(t_{P_{2}}{ }^{k}+c_{P_{1}}{ }^{k}+c_{P_{2}}{ }^{k}\right)\right)+\ldots
$$

In this case, task queue is as follows:

$\operatorname{Run}\left(q_{k}, t_{l_{k}}\right)=$

$$
\begin{aligned}
& P_{1}{ }^{k}\left(0, d_{P_{1}}{ }^{k}-c_{P_{1}}{ }^{k}, v_{P_{1}}\left(t_{P_{1}}{ }^{k}+c_{P_{1}}{ }^{k}\right)\right), \\
& P_{2}{ }^{k}\left(c_{P_{2}}{ }^{k}, d_{P_{2}}{ }^{k}-c_{P_{1}}{ }^{k}, v_{P_{2}}\left(t_{P_{2}}{ }^{k}+d_{l_{k}}\right)\right), \\
& \ldots \ldots \ldots ., \\
& \left.P_{n}{ }^{k}\left(c_{P_{n}}{ }^{k}, d_{P_{n}}{ }^{k}, v_{P_{n}}\left(t_{P_{n}}{ }^{k}+d_{l_{k}}\right)\right)\right] .
\end{aligned}
$$

Here $t_{l_{k}}=d_{l_{k}}$.

Therefore we compute the value as follows:

(c) $c_{P_{1}}{ }^{k}>d_{l_{k}}$ :

$$
\left.v_{P_{1}}\left(t_{P_{1}}^{k}+c_{P_{1}}{ }^{k}\right)\right)
$$

In this case, task queue is as follows:

As $\operatorname{Run}\left(q_{k}, t_{l_{k}}\right)=[$

$$
\begin{aligned}
& P_{1}{ }^{k}\left(c_{P_{1}}{ }^{k}-d_{l_{k}}, d_{P_{1}}{ }^{k}-d_{l_{k}}, v_{P_{1}}\left(t_{P_{1}}{ }^{k}+d_{l_{k}}\right),\right. \\
& P_{2}{ }^{k}\left(c_{P_{2}}{ }^{k}, d_{P_{2}}{ }^{k}-d_{l_{k}}, v_{P_{2}}\left(t_{P_{2}}{ }^{k}+d_{l_{k}}\right)\right), \\
& \ldots \ldots \ldots ., \\
& \left.P_{n}{ }^{k}\left(c_{P_{n}}{ }^{k}, d_{P_{n}}{ }^{k}-d_{l_{k}}, v_{P_{n}}\left(t_{P_{n}}{ }^{k}+d_{l_{k}}\right)\right)\right],
\end{aligned}
$$

we can not compute the value.

Here $t_{l_{k}}=d_{l_{k}}$.

4. $t_{l_{k}} \geq d_{l_{k}}$ :

In this case, although $c_{P_{i}}{ }^{k}$ is less or greater than $d_{l_{k}}$, task queue is as follows:

$\operatorname{Run}\left(q_{k}, t_{l_{k}}\right)=[$

$$
\begin{aligned}
& P_{1}{ }^{k}\left(0, d_{P_{1}}{ }^{k}-c_{P_{1}}{ }^{k}, v_{P_{1}}\left(t_{P_{1}}{ }^{k}+c_{P_{1}}{ }^{k}\right)\right), \\
& P_{2}{ }^{k}\left(0, d_{P_{2}}{ }^{k}-\left(c_{P_{1}}{ }^{k}+c_{P_{2}}{ }^{k}\right), v_{P_{2}}\left(t_{P_{2}}{ }^{k}+c_{P_{1}}{ }^{k}+c_{P_{2}}{ }^{k}\right)\right), \\
& \left.\ldots \ldots \cdots \cdot{ }^{k}{ }^{k}{ }^{k}\left(0, d_{P_{n}}{ }^{k},-\left(c_{P_{1}}{ }^{k}+\ldots+c_{P_{n}}{ }^{k}\right), v_{P_{n}}\left(t_{P_{n}}{ }^{k}+c_{P_{1}}{ }^{k}+\ldots+c_{P_{n}}{ }^{k}\right)\right)\right] .
\end{aligned}
$$

Here $t_{l_{k}}=c_{P_{1}}{ }^{k}+\ldots+c_{P_{n}}{ }^{k}$.

Therefore we compute the value as follows:

$$
\left.\left.v_{P_{1}}\left(t_{P_{1}}{ }^{k}+c_{P_{1}}{ }^{k}\right)\right)+\ldots+v_{P_{n}}\left(t_{P_{n}}{ }^{k}+c_{P_{1}}{ }^{k}+\ldots+c_{P_{n}}{ }^{k}\right)\right)
$$


5. $t_{l_{k}}>d_{l_{k}}$ :

In this case, although $c_{P_{i}}{ }^{k}$ is less or greater than $d_{l_{k}}$, task queue is as follows:

$\operatorname{Run}\left(q_{k}, t_{l_{k}}\right)=[$

$$
\begin{aligned}
& \quad P_{1}^{k}\left(0, d_{P_{1}}{ }^{k}-c_{P_{1}}{ }^{k}, v_{P_{1}}\left(t_{P_{1}}{ }^{k}+c_{P_{1}}{ }^{k}\right)\right), \\
& \quad P_{2}{ }^{k}\left(0, d_{P_{2}}{ }^{k}-\left(c_{P_{1}}{ }^{k}+c_{P_{2}}{ }^{k}\right), v_{P_{2}}\left(t_{P_{2}}{ }^{k}+c_{P_{1}}{ }^{k}+c_{P_{2}}{ }^{k}\right)\right) \text {, } \\
& \left.\left.\left.\quad \ldots \ldots \ldots,{ }^{k} \ldots c_{P_{n}}{ }^{k}\right)\right)\right] . \\
& \quad P_{n}{ }^{k}\left(0, d_{P_{n}}{ }^{k},-\left(c_{P_{1}}{ }^{k}+\ldots+c_{P_{n}}{ }^{k}\right), v_{P_{n}}\left(t_{P_{n}}{ }^{k}+c_{P_{1}}{ }^{k}+c_{P_{1}}{ }^{k}+\ldots+c_{P_{n}}{ }^{k}\right. \text {. }\right. \\
& \text { Therefore we compute the value as follows: }
\end{aligned}
$$

$$
\left.\left.v_{P_{1}}\left(t_{P_{1}}{ }^{k}+c_{P_{1}}{ }^{k}\right)\right)+\ldots+v_{P_{2}}\left(t_{P_{2}}{ }^{k}+c_{P_{1}}{ }^{k}+\ldots+c_{P_{n}}{ }^{k}\right)\right)
$$

\section{Discrete transition:}

After delay transition, as $l \stackrel{g, a, r}{\longrightarrow} m$ and $u \models g$ hold true, the following transition occurs:

$$
(l, u, q) \stackrel{g, a, r}{\longrightarrow} S c h(m, u[r \mapsto 0], \mathbf{S c h}(M(m):: q)),
$$

where $\mathbf{S c h}(M(m):: q)$ can be obtained by adding the task in node $m$.

For each deterministic behavior, by repeating 1. and 2. from an initial node, we can compute $\sum_{i=1}^{n} v_{P_{i}}\left(t_{P_{i}}{ }^{k}\right)$.

Therefore, we can get the sum of values for each deterministic behavior.

\section{Conclusion}

In this paper, we develop an automata-theoretic performance analysis method of soft real-time systems by extending the verification method of schedulability of hard real-time systems using utility functions. In our proposed method, task is characterized as the execution time, the deadline and the utility function. Moreover, performance analysis can be transformed to a brute force search for timed computation tree of timed automata and a calculation of the value of every state. We have implemented an automata-theoretic performance analyzer on Sun Blade2000. Now, we are going to apply our proposed method into practical problems.

\section{References}

1. M. Joseph, editor. Real-time Systems: Specification, Verification and Analysis. Prentics-Hall, 1996.

2. Jane W. S. Liu. Real-Time Systems. Prentics-Hall,2000.

3. Giorgio C. Buttazzo. Hard Real-Time Computing Systems : Predictable Scheduling Algorithms and Applications. Kluwer, 1997.

4. R. Alur, D.L. Dill. A theory of timed automata. TCS,Vol. 126, pp.183-235, 1994.

5. R. Alur, C. Courcoubetis, D.L. Dill. Model-Checking in Dense Real-Time. Information and Computation, Vol. 104, pp. 2-34, 1993.

6. T. A. Henzinger, X. Nicollin, J. Sifakis, S. Yovine. Symbolic model checking for real-time systems. Information and Computation, Vol. 111, pp. 193-244, 1994. 
7. C. Ericsson, A. Wall, Wang Yi. Timed Automata as Task Models for Event driven Systems. Proc. of RTSCA 99, pp.182-189, IEEE CS, 1999.

8. E. Fersman, P. Pettersson, Wang Yi. Timed Automata with Asynchrounous Processes: Schedulability and Decidability. LNCS 2280, pp.67-82, 2002.

9. J. McManis, P. Varaiya. Suspension automata: A decidable class of hybrid automata. LNCS 818, pp.105-117, 1994.

10. J. C. Corbett. Modeling and analysis of real-time Ada tasking programs. Proc. of RTSS, pp. 132-141, IEEE CS, 1994.

11. F. Cassez, F. Laroussinie. Model-checking for hybrid systems by quotienting and constraints solving. LNCS 1855, pp. 373-388, 2000.

12. R. Alur, C. Coucoubetis, N. Halbwachs, et-al. The algorithmic analysis of hybrid systems. TCS, Vol.138, pp.3-34, 1995.

13. P. Bouyer, C. Dufourd, E. Fleury, and A. Petit. Expressiveness of updatable timed automata. LNCS 1893, pp.232-242, 2000.

14. E. D. Jensen, C. D. Locke and H. Tokuda. A time-driven scheduling model for realtime operating systems. Proc. of RTSS, pp.112-122, 1985.

15. B. Kao, H. Garcia-Molina, and B. Adelberg. On building distributed soft real-time systems. Proc. of WPDRTS, pp.13-19, 1995.

16. K.M. Kavi, H.Y. Youn, B. Shirazi, A.R. Hurson. A Performability Model for Soft Real-Time Systems. Proc. of HICSS, pp.571-579, 1994.

17. M. K. Gardner. Probabilstic Analysis and Scheduling of Critical Soft Real-time Systems. PhD thesis, University of Illinois, Urbana, Illinois, 1999.

18. H. Hansson and B. Jonsson. A calculus for communicating systems with time and probabilities. Proc. of RTSS, pp.278-287, 1990.

19. R. Alur, C. Courcoubetis, D.L. Dill. Model-checking for probabilistic real-time systems. LNCS 510, pp. 115-136, 1991.

20. M. Kwiatkowska, G. Norman, R. Segala and J. Sproston. Automatic Verification of Real-Time Systems With Discrete Probability Distributions. LNCS 1601, pp. 75-95, 1999

21. P.R. D'Argenio, J.-P. Katoen, E. Brinksma. Specification and Analysis of Soft RealTime Systems: Quantity and Quality. Proc. of RTSS, pp. 104-114, 1999.

22. K. Tindell. Fixed-Priority Scheduling of Hard Real-Time Systems. PhD thesis, University of York, UK, 1994.

23. D.L. Dill. Timing assumptions and veri cation of finite-state concurrent systems. LNCS 40\%, pp.197-212, 1989.

24. S. Yamane, K. Nakamura. Symbolic Model-Checking Method Based on Approximations and BDDs for Real-Time Systems. LNCS 1281, pp.562-582, 1997. 